\title{
ABSENCE OF GOLDSTINO DECOUPLING IN HIERARCHICAL SUPERUNIFIED MODELS
}

\author{
M.B. EINHORN and D.R.T. JONES \\ Randall Laboratory of Physics, University of Michigan, Ann Arbor, MI 48109, USA
}

Received 16 May 1983

\begin{abstract}
It is shown that there exists a class of hierarchical supersymmetric unified models in which the Goldstino field does not decouple from the light states. We illustrate this with an $\mathrm{SU}_{N}$ model. The phenomenology and cosmology of such models is discussed.
\end{abstract}

In supersymmetric gauge theories with O'Raifeartaigh-type supersymmetry (SUSY) breaking it is generally the case that the vacuum expectation value (VEV) of the scalar super partner of the Goldstino field is not determined in the tree approximation [1]. It was suggested by Witten that the vacuum expectation value (VEV), $\langle G\rangle$ might be determined by radiative corrections to be much larger than any mass parameter present in the original superpotential [2]. Such mass parameters we will denote by $m$. Subsequent analysis showed that $\langle G\rangle$ is indeed determined from a renormalization-group improved, one-loop calculation of the effective potential ${ }^{\neq 1}[3-5]$. While in Witten's original model a degree of fine tuning is required [6] to produce $G \gg m$, the possibility remains that in general a large hierarchy ("inverted hierarchy") may be produced in this way.

In Witten's original model it is the case that, if $\langle G\rangle \gg m$, then only supermultiplets of mass $\mathrm{O}(\langle\mathrm{G}\rangle)$ suffer SUSY breaking in the tree approximation; states of mass $m$ or zero remain in degenerate supermultiplets, and gain SUSY breaking corrections of $\mathrm{O}\left(\mathrm{m}^{2} /\langle\mathrm{G}\rangle\right)$ through radiative corrections. Thus one is led to identify $\mathrm{m}^{2} /\langle\mathrm{G}\rangle$ with the weak scale, $m$ with some intermediate scale $\mathrm{O}\left(10^{11} \mathrm{GeV}\right)$ and $\langle\mathrm{G}\rangle$ with some grand unification (or Planck?) scale $[5,7]$.

This decoupling phenomenon is in fact perfectly general in O'Raifeartaigh-type models as long as $\mathrm{G}$ is

${ }^{\ddagger 1}$ Note that $\beta_{\lambda}$ and $\beta_{\lambda}{ }^{\prime}$ in ref. [5] disagree by a factor of two from those in refs. $[3,4]$. the only field whose VEV is undetermined in the tree approximation [1]. This is easily seen by expressing the superpotential of a general model in terms of $G$ and other fields $\phi_{i}$ of zero VEV. One finds (schematically)

$W=-t \mathrm{G}+a_{i j} \phi_{i} \phi_{j} \mathrm{G}+m_{i j} \phi_{i} \phi_{j}+C_{i j k} \phi_{i} \phi_{j} \phi_{k}$

From eq. (1) it can be seen that if $G$ develops a large VEV, then supersymmetric mass terms of $O(G)$ occur for the fields which couple to the Goldstino superfield; which is to say that in the limit $\langle G\rangle \gg m$, the theory becomes supersymmetric. It is evident, however, that the argument fails in general if one or more of the fields $\phi_{i}$ also has an arbitrary VEV, since such a VEV could cancel the large mass terms due to $\langle G\rangle$ leaving a light field still coupled to the Goldstino.

At this point such a development seems to require fine tuning, but, as we show below, that is not in general the case. Our main purpose here is to draw attention to the existence of a class of models in which, while the inverted hierarchy can occur, the SUSY decoupling is incomplete and so the necessity for the introduction of an intermediate scale rendered suspect. We do this by examining a simple $\mathrm{SU}_{N}$ model. In the model, the direction of the breaking in $\mathrm{SU}_{N}$ space is not fully determined in the tree approximation, and it turns out that whether or not decoupling occurs depends on the symmetry breaking direction chosen by the radiative corrections.

Consider the superpotential 
$W=\lambda_{1} H_{i} Y_{j}^{i} K^{j}+\lambda_{2} X\left(H_{i} K^{i}-m^{2}\right)$,

where $X, Y, H, K$ are respectively singlet, adjoint, $\bar{N}$ and $N$ representations of $\mathrm{SU}_{N}$.

Up to gauge transformations the effective potential is minimized in the tree approximation by the following scalar VEVs:

$\langle H\rangle=\langle K\rangle=(0 \ldots 00 h)^{\mathrm{T}}$,

$\langle X\rangle=\left(-\lambda_{1} / \lambda_{2}\right)\left\langle Y_{N N}\right\rangle$,

$\langle Y\rangle \equiv \Delta$ arbitrary real diagonal,

where

$h^{2}=\frac{\lambda_{2}^{2} m^{2}}{\lambda_{2}^{2}+[(N-1) / N] \lambda_{1}^{2}}$.

At the minimum the vacuum energy $V_{0}$ is given by

$V_{0}=\frac{m^{4} \lambda_{1}^{2} \lambda_{2}^{2}}{\lambda_{2}^{2}+[(N-1) / N] \lambda_{1}^{2}}$.

So SUSY is broken at the scale $m$.

Now let

$Y \rightarrow \Delta+Y, \quad X \rightarrow\langle X\rangle+X, \quad H \rightarrow\langle H\rangle+H$,

$K \rightarrow\langle K\rangle+K$

The superpotential becomes (in terms of shifted fields)

$$
\begin{aligned}
& W=-\left(\frac{N-1}{N}\right)^{1 / 2} m^{2} \frac{\lambda_{1} \lambda_{2}}{r} G+h r A\left(H_{N}+K_{N}\right) \\
& +\lambda_{1} h\left[H_{1}\left(Y_{1}-\mathrm{i} Y_{2}\right)+H_{2}\left(Y_{4}-\mathrm{i} Y_{5}\right) \ldots\right. \\
& +H_{N-1}\left(Y_{(N-1)^{2}}-\mathrm{i} Y_{(N-1)^{2}+1}\right)+K_{1}\left(Y_{1}+\mathrm{i} Y_{2}\right) \\
& \left.+K_{2}\left(Y_{1}+\mathrm{i} Y_{2}\right) \ldots+K_{N-1}\left(Y_{(N-1)^{2}}+\mathrm{i} Y_{(N-1)^{2}+1}\right)\right] \\
& +\lambda_{1}\left[\left(\Delta_{1}-\Delta_{N}\right) H_{1} K_{1}+\left(\Delta_{2}-\Delta_{N}\right) H_{2} K_{2} \ldots\right. \\
& \left.+\left(\Delta_{N-1}-\Delta_{N}\right) H_{N-1} K_{N-1}\right] \\
& +\frac{r N}{N-1} \sin \alpha \cos \alpha G\left(H_{1} K_{1}+H_{2} K_{2}+\ldots H_{N-1} K_{N-1}\right) \\
& + \text { cubic terms not involving } G \text {, }
\end{aligned}
$$

where

$$
r^{2}=\lambda_{2}^{2}+\frac{N-1}{N} \lambda_{1}^{2}, \quad \sin \alpha=\frac{\lambda_{2}}{r},
$$

$G=\cos \alpha X+\sin \alpha Y_{N^{2}-1}$

$A=\sin \alpha X-\cos \alpha Y_{N^{2}-1}$.

Now suppose Witten's inverted hierarchy idea is realized, i.e. some at least of the $\Delta_{i} \gg m$. If it should turn out that $\Delta_{i}-\Delta_{N} \gg m$ for all $i \neq N$, then the Goldstino couples only to those fields which have large masses, and so decoupling occurs. However, for any $i$ such that $\Delta_{i}-\Delta_{N}=\mathrm{O}(m)$, there arises a supermultiplet of mass $\mathrm{O}(\mathrm{m})$ to which $\mathrm{G}$ couples, that is, it has SUSY splittings of $\mathrm{O}(m)$ and avoids decoupling $\$ 2$. Consider, for example, the case $N=5$, and suppose that radiative corrections determine $\Delta \propto(222-3-3)$. Since $\Delta_{4}=\Delta_{5}$, decoupling does not occur; the superpotential appropriate to describe phenomena at energies $\ll \Delta$ is

$W=\lambda_{1}^{*} H_{i} Y_{j}^{i} K^{j}+\lambda_{2}^{*} X\left(H_{i} K^{i}-m^{2}\right)$,

where now $i=1,2$ only; $\lambda_{1}^{*}$ and $\lambda_{2}^{*}$ are the appropriately renormalized couplings ${ }^{\neq 3}$. Thus, we would have the desirable symmetry breaking sequence

$$
\mathrm{SU}_{5} \stackrel{\Delta}{\rightarrow} \mathrm{SU}_{3} \times \mathrm{SU}_{2} \times \mathrm{U}_{1} \underset{\substack{\text { sUSY } \\ \text { breaking }}}{\stackrel{m}{\longrightarrow}} \mathrm{SU}_{3} \times \mathrm{U}_{1}
$$

and the nucleus of a possibly phenomenologically acceptable model. Matter generations $(\overline{5}+10)$ may be added in the usual way; trilinear couplings to $H$ and $K$ do not disturb the tree vacuum (3). The hierarchy is natural as can be seen by examining the limit $m \rightarrow 0$; in that limit SUSY returns but $\Delta$ remains fixed by dimensional transmutation as discussed at length in ref. [3].

Then the SUSY non-renormalization theorems [8] assure us that no light state is lifted up to $O(\Delta)$. There

\$2 Although there remain fields, such as $H_{N}, K_{N}$, which still decouple in tree approximation, these supermultiplets acquire splittings of $\mathrm{O}(\mathrm{m})$ from radiative corrections.

$\$ 3$ In ref. [1] it is claimed that in models of the type considered here there is no choice of renormalization mass $\mu$ for which perturbative calculations are reliable because of the presence of both $\ln ((\mathrm{G}) / \mu)$ and $\ln (\mathrm{m} / \mu)$ terms in the one loop effective potential. We shall argue elsewhere that this is not true in general; in the model considered here, for example, $\mathrm{S} \operatorname{Tr} M^{4}$ vanishes separately for light and heavy fields at the minimum, and this ensures absence of large logarithms for that particular choice of $\mu$. A generalization of the renormalization group analysis of ref. [3] to this type of theory is in preparation. 
are additional nice features; it is clear from (7), for example, that the Higgs triplet $(3,1)$ states automatically get masses of $O(\Delta)$. Then the unification mass $M_{\mathrm{X}}$ and $\sin ^{2} \theta_{\mathrm{w}}$ can be calculated from the running couplings in the usual way [9]. One finds $M_{\mathrm{X}} \sim 10^{22}$ $\mathrm{GeV}$ and $\sin ^{2} \theta_{\mathrm{w}} \sim 0.23$. One can add $\mathrm{SU}_{3} \times \mathrm{SU}_{2}$ singlet representations to reduce the value of $M_{\mathrm{x}}$ without disturbing the prediction for $\sin ^{2} \theta_{w}$. There are also problems, however, in that there is a massless octet supermultiplet, and also gluinos do not acquire perturbative masses. These problems might be resolved by introducing a hidden sector coupled via $N=1$ supergravity. We do not pursue these considerations here, however, since we have not shown that the $\mathrm{SU}_{5}$ breaking is in the desired direction. The resolution of this question requires a generalization of the methods of ref. [3] to the case of several undetermined fields; this is under study. Another problem with the model is that, as in Witten's original model [6], a fine tuning of the renormalized coupling is required to produce a large hierarchy. One might hope to finesse this problem by taking trajectories within the domain of attraction of the origin in coupling constant space, when the scale $\Delta$ is determined by gravitational effects [10]. However unfortunately in this case that scale is determined to be $\Delta \propto \operatorname{diag}(1111-4)$.

Models of the Witten-type present certain cosmological difficulties. It has been emphasised [11] that, because of decoupling, the rate at which the Higgs field radiates as it relaxes to the minimum of the effective potential is too slow to reheat the universe. Consequently, the baryon density, diluted by the inflationary expansion, remains incredibly small. This problem of reheating is neatly resolved by models of the sort considered herein which avoid decoupling. However, the model developed in this paper also fails because the universe is reheated only to the weak scale, not the GUT scale. To fulfill this cosmological constraint, one requires a geometric hierarchy without decoupling, which seems in principle possible. Then the universe will reheat to the intermediate scale, whereupon baryosynthesis can recur. It would be of interest to develop such a model with a phenomenologically acceptable breaking pattern.
Coupling to supergravity complicates the discussion further, since all scalars receive masses of order the gravitino mass, $\tilde{m}_{\mathrm{g}}$, if $N=1$ supergravity is any guide. For small $\widetilde{m}_{\mathrm{g}}$, a local minimum remains but there are examples in which there arises a global supersymmetric minimum elsewhere. So there is no assurance of stability to gravitational effects. For large $\tilde{m}_{\mathrm{g}}$, the preceding analysis certainly requires modification.

In summary, we have illustrated that supersymmetric decoupling depends on the symmetry-breaking pattern of a superunified model. It remains to be seen whether this can be incorporated into a phenomenologically and cosmologically acceptable model.

We would like to thank S. Raby for discussions. This work was supported in part by the Department of Energy.

\section{References}

[1] See, for example, the recent discussion by J. Polchinski, Phys. Rev. D27 (1983) 1320;

B. Zumino, LBL-13691 (Dec., 1982).

[2] E. Witten, Phys. Lett. 105B (1981) 267.

[3] M.B. Einhorn and D.R.T. Jones, Nucl. Phys. B211 (1983) 29.

[4] II. Yamagishi, Princeton preprint (1982).

[5] T. Banks and V. Kaplunovsky, Nucl. Phys. B211 (1983) 529.

[6] L. Hall and I. Hinchliffe, Phys. Lett. 119B (1982) 128.

[7] J. Polchinski and L. Susskind, Phys. Rev. D26 (1982) 3661 ;

S. Dimopoulos and S. Raby, Nucl. Phys. B., to be published.

[8] M.T. Grisaru, M. Rocek and W. Siegel, Nucl. Phys. B159 (1979) 429;

see also M.T. Grisaru, four lectures on supergraphs, ICTP Spring School on Supergravity (Trieste, April 1981), and references therein.

[9] See, e.g., M.B. Einhorn and D.R.T. Jones, Nucl. Phys. $B 196$ (1982) 475, and references therein.

[10] B. Ovrut and S. Raby, Phys. Lett. 121B (1983) 381.

[11] A. Albrecht et al., submitted to third Marcel Grossman Meeting on Recent developments in general relativity (Shanghai, China, Aug., 1982);

J. Preskill, M.B. Wise and F. Wilczek, Harvard preprint HUTP-82/A048. 